\title{
Factors Affecting Lymph Node Yield in Surgically Resected Colorectal Cancer Specimens
}

\author{
Deepa Nagarajan ${ }^{1 *}$, Rupa Ashish Jain², Anita Bharat Shah ${ }^{3}$, Rajesh Munde ${ }^{3}$ and Mita Y Shah ${ }^{3}$ \\ ${ }^{1}$ Department of Pathology, Lilavati Hospital and Research Centre \\ ${ }^{2}$ Department of Laboratory medicine, Saifee Hospital \\ ${ }^{3}$ Department of Histopathology, Saifee Hospital
}

\begin{abstract}
Background: Currently, the CAP protocol mandates evaluation of $\geq 12$ Lymph nodes as a quality indicator for the adequacy of pathologic examination of colorectal cancer resection specimens. Aim: To identify factors that may influence the lymph node yield in colorectal cancer specimens and to compare with the relevant publications.

Methods: The retrospective study of seventy patients with loco-regional colorectal adenocarcinomas treated by standard surgical resection from April 2015- April 2017 was included. All cases with inadequate lymph nodes had been re-grossed by another pathologist. Variables like age, gender, primary site, type of surgery, specimen length, tumour size, grade and stage, neoadjuvant therapy and tumour site perforation were evaluated for their impact on the average total number of nodes examined.

Results: Out of seventy, eleven [15.71\%] patients had inadequate mean nodal yield [MNY]. Of these eleven patients, MNY was greater in males [6.6] than in females [6.4]. MNY was lesser in patients with age $>50$ years [5.71] than patients $\leq 50$ years [8]. The yield increased exponentially with increasing tumour stage and tumour size. Yield was higher in tumours with perforation. Specimens longer than $20 \mathrm{~cm}$ had a higher yield [7.29] than in shorter specimens [5.25]. The yield was lesser when tumour is located more distally [APR:4.5 and AR \&sigmoid colectomy:7.7]. Seven patients had taken neoadjuvant therapy [63.6\%] of whom, six had moderately differentiated adenocarcinoma \& one had no tumour.
\end{abstract}

Conclusion: Factors like neoadjuvant therapy, age \& gender of the patient, type of surgery, length of the specimen, tumour size, grade, stage, site \& perforation, affect the MNY in colorectal cancers.

Keywords: Colorectal cancer, Lymph node, Nodal yield

\section{Introduction}

The presence of at least 12 lymph nodes in a surgical resection is among the key quality measures for colon cancer care in the United States, as listed by the National Quality Forum in the year 2007. ${ }^{[1]}$

Examination of at least 12 lymph nodes is recommended by tumour node metastasis [TNM] staging system, developed, and maintained by the Union for International Cancer Control [UICC] and American Joint Committee on Cancer [AJCC] for adequate colorectal cancer staging. ${ }^{[1]}$ Cases with inadequate lymph node include ones with less than twelve.

Adequate numbers of lymph nodes are essential for accurate staging. Lymph node metastasis affects the prognosis and helps in the management of the patient, particularly the need for adjuvant chemotherapy. ${ }^{[2]}$

Lymph node yield is affected by numerous factors related to the patient, the tumour, surgical and histopathological practice. ${ }^{[2]}$
The accuracy and predictive value of stage II are directly depending on two modifiable factors:

1) Surgical technique in dissecting all regional nodes and

2) Pathologic examination of the resection specimen in identifying and harvesting all regional lymph nodes for microscopic assessment. ${ }^{[1]}$

Despite recommendations, inadequate lymph nodes evaluation is common. Inadequate Lymph nodes staging in colorectal cancer can potentially lead to under staging patients and insufficient adjuvant treatment. These cases should be re-grossed by another pathologist so that additional lymph nodes are found, which leads to correct staging.

Several studies state multiple factors associated with better and higher lymph node yield such as patient characteristics (age, sex, ethnicity), gross characteristics (tumour size and location), microscopic tumour characteristics (differentiation grade, tumour invasion) and surgeon factor, but all show debatable results. 
This study is aimed to review the various factors that influence lymph node yield in colorectal cancer cases and to compare with the relevant published data.

\section{Materials and Methods}

In this retrospective study, we included 70 patients aged between 26 and 86 years who had locoregional primary invasive colorectal adenocarcinoma treated at our Institution between April 2015 and April 2017.

All patients were staged according to the 7 th edition of the AJCC TNM staging system. ${ }^{[1,3]}$

Inclusion criteria included patient with locoregional primary colorectal adenocarcinoma with the primary site being sigmoid colon, rectosigmoid junction and rectum. Patients with and without neoadjuvant therapy were included.

Exclusion criteria included patients presenting with pathologies other than adenocarcinoma, patients with metastatic disease in the colon, patients who had undergone right colectomy, total colectomy, or palliative surgery.

In this study, a comprehensive literature review using the search terms of "lymph-node" and "colorectal" or "colon" or "rectum" or "rectal" was done to find out the major related studies for discussing the manuscript. Articles in non-English language or with unavailable full text were excluded. Informative abstracts were included.

The mean number of total lymph nodes examined was initially calculated. Subsequently, the percentage of patients who had at least 12 nodes examined and the percentage of patients who had less than 12 lymph nodes in their pathologic report were determined.

All potential tumour (anatomical site, tumour size, grade, bowel perforation and the number of involved lymph nodes), patient (age and sex) and treatment (type of surgery and neoadjuvant treatments) characteristics were evaluated for their impact on the average total number of lymph node examined.

\section{Results}

All patients with primary colon cancer were treated with standard curative surgical resection. A total of 1548 lymph nodes were identified in the 70-resection specimen with a median of 22.11 lymph nodes per patient (range 0-72 lymph nodes) (table 1)

Lymph node yield was adequate in 59 patients (84.2\%) and 11 patients $(15.71 \%)$ had inadequate yield. Thirty-two (84.21\%) male patients had adequate yield, 6 (15.79\%) had inadequate yield. Twenty-seven $(84.38 \%)$ female patients had adequate lymph node yield, whereas 5(15.62\%) patients had inadequate yield.
Twenty-three $(32.85 \%)$ patients were $\leq 50$ years. Amongst them, 19 patients $(82.6 \% \%)$ had adequate yield and 4 patients $(17.4 \%)$ had inadequate yield. Forty-seven patients $(67.14 \%)$ were more than 50 years old. Out of 47 patients, 40 patients $(85.10 \%)$ had adequate yield, whereas 7 patients $(14.9 \%)$ had inadequate yield.

Thirty patients $(42.85 \%)$ had tumour location in the sigmoid colon and rectosigmoid junction. Of them, twentyseven patients $(90 \%)$ had adequate yield and three (10\%) had inadequate yield. Of 40 patients $(57.14 \%)$ with tumour location in the rectum, 32 patients $(80 \%)$ had adequate yield and 8 patients $(20 \%)$ had inadequate yield.

Fifty-four patients (77.14\%) undergone sigmoid colectomy and anterior resection surgery. Of them, forty-seven patients $(87.04 \%)$ had adequate yield and seven $(12.96 \%)$ had inadequate yield. Of sixteen patients $(22.85 \%)$ who had undergone APR surgery, 12 patients (75\%) had adequate yield and 4 patients (25\%) patients had inadequate yield.

There were two cases each in pT0, pTIS and pT1 respectively $(2.86 \%$ each). Each stage had 1 case with adequate and an inadequate yield (50\% each). Fourteen patients $(20 \%)$ had stage pT2, with 11 patients $(78.57 \%)$ having adequate yield and $3(21.43 \%)$ having inadequate yield. Forty patients (57.14\%) had stage pT3, of which, 37 patients $(92.5 \%)$ had adequate yield and $3(7.5 \%)$ patients had inadequate yield. Ten patients $(14.29 \%)$ had stage pT4; eight patients $(80 \%)$ amongst them had adequate yield and 2 patients $(20 \%)$ had inadequate yield.

Thirty-three patients (47.14\%) had tumour size $\leq 5 \mathrm{~cm}$. Of them, twenty-seven patients $(81.82 \%)$ had adequate yield and 6 patients $(18.18 \%)$ had inadequate yield. Thirtyseven patients $(52.85 \%)$ had tumour size $>5 \mathrm{~cm}, 32$ patients $(86.49 \%)$ amongst them had adequate yield and 5 patients (13.51\%) patients had inadequate yield.

Fifty-five patients (78.57\%) had low-grade tumour (54 patients had moderately differentiated adenocarcinoma + 1 had well-differentiated adenocarcinoma). Amongst them, 46 patients $(83.64 \%)$ had adequate yield and 9 patients $(16.36 \%)$ had inadequate yield.

Thirteen patients (18.57\%) had high-grade tumour (poorly differentiated adenocarcinoma). Of them, 12 patients $(92.30 \%)$ had adequate yield and one $(7.7 \%)$ had inadequate yield. Two patients $(1.43 \%$ ) had no tumour (no residual tumour post neoadjuvant therapy).

Tumour site perforation was present in 10 patients (14.28\%). Of them, eight (11.42\%) had adequate yield and two $(2.86 \%)$ had inadequate yield. Sixty patients $(85.72 \%)$ had intact tumour site wall, without perforation. Of them, 9 patients $(12.86 \%)$ had inadequate yield. 
Twenty-one patients out of these seventy received neoadjuvant chemoradiation followed by curative-intent surgery. All patients receiving neoadjuvant chemoradiation underwent standard curative surgery with at least 4-6 weeks interval. Twenty-one patients (30\%) had taken neoadjuvant therapy. Of them, seven patients $(33.33 \%)$ had inadequate yield (Table 2).

In eleven patients with inadequate lymph node yield, male patients had MNY of 6.6 and female patients had MNY 6.4. Patients with age $>50$ years had a lesser nodal yield of 5.71 compared to patients with age less than or equal to 50 years with a nodal yield of 8 .

Patients who had undergone APR surgery had a MNY of 4.5 and patients who had undergone AR surgery \& rectosigmoid colectomy had a yield of 7.7. Yield increased with tumour stage $(6,7.3,7.6$ and 10 with pT1, pT2, pT3 and pT4 stages). The yield increased with tumour size (8 with tumour with $>5 \mathrm{~cm}$ and 5.3 with tumour with less than or equal to $5 \mathrm{~cm}$ ). Nine patients had moderately differentiated adenocarcinoma (low-grade tumour), one had poorly differentiated adenocarcinoma [high-grade tumour] and one patient had no residual tumour. The yield was higher in tumour's with perforation (mean lymph nodal yield:10) than in tumours without perforation (MNY: 5.77). The yield was higher in tumours with specimen length more or equal to $20 \mathrm{~cm}$ (MLY: 7.29) than in tumours with lesser than $20 \mathrm{~cm}$ length [mean lymph nodal yield: 5.25 ].

Of these eleven patients, seven patients $(63.6 \%)$ had taken neoadjuvant therapy (MNY: 6.7\%). Amongst them, six patients had moderately differentiated adenocarcinoma \& one had no residual tumour.

Table 1: Case distribution and analysis of various factors on MLY along with ' $p$ ' value calculation:

\begin{tabular}{|c|c|c|c|c|}
\hline Factors & & Adequate cases & Inadequate cases & p'value \\
\hline \multicolumn{5}{|c|}{ 1.Gender: } \\
\hline & Male & 32 & 6 & 0.77 \\
\hline & Female & 27 & 5 & \\
\hline \multicolumn{5}{|l|}{ 2.Age: } \\
\hline & $\leq 50$ years & 19 & 4 & 0.74 \\
\hline & $>50$ years & 40 & 7 & \\
\hline \multicolumn{5}{|c|}{ 3.Primary site: } \\
\hline & Rectum & 32 & 8 & 0.33 \\
\hline & RSJ and Sigmoid colon & 27 & 3 & \\
\hline \multicolumn{5}{|c|}{ 4. Type of surgery: } \\
\hline & APR & 12 & 4 & 0.25 \\
\hline & AR \& SC & 47 & 7 & \\
\hline \multicolumn{5}{|c|}{ 5.Specimen length: } \\
\hline & $\leq 20 \mathrm{~cm}$ & 45 & 4 & 0.008 \\
\hline & $>20 \mathrm{~cm}$ & 14 & 7 & \\
\hline \multicolumn{5}{|c|}{ 6.Primary tumor stage } \\
\hline & T0 & 1 & 1 & \\
\hline & TIS & 1 & 1 & \\
\hline & T1 & 1 & 1 & \\
\hline & T2 & 11 & 3 & \\
\hline & T3 & 37 & 3 & \\
\hline & T4 & 8 & 2 & \\
\hline \multicolumn{5}{|c|}{ 7.TUMOR SIZE } \\
\hline & $</=5 \mathrm{~cm}$ & 27 & 6 & 0.59 \\
\hline & $>5 \mathrm{~cm}$ & 32 & 5 & \\
\hline \multicolumn{5}{|c|}{ 8.Tumor Grade } \\
\hline & No Residual tumor & 1 & 1 & \\
\hline & Low Grade & 46 & 9 & \\
\hline & High Grade & 12 & 1 & \\
\hline
\end{tabular}




\begin{tabular}{|l|l|l|l|l|}
\hline Factors & Adequate cases & Inadequate cases & p' value \\
\hline 9.Tumor site perforation & & & \\
\hline & Absent & 51 & 9 & 0.65 \\
\hline & Present & 8 & 2 & \\
\hline 10.Neoadjuvant therapy & & & 0.013 \\
\hline & Yes & 14 & 7 & \\
\hline & No & 45 & 4 & \\
\hline
\end{tabular}

TABLE 2: Factors affecting the Lymph node yield in specimen with inadequate lymph node yield are as under:

\begin{tabular}{|c|c|c|}
\hline Factors & Number of patients & Inadequate Lymph nodes yield \\
\hline \multicolumn{3}{|l|}{ 1.Gender } \\
\hline Male & 6 & 6.6 \\
\hline Female & 5 & 6.4 \\
\hline \multicolumn{3}{|l|}{ 2. Age } \\
\hline$\leq 50$ years & 4 & 8 \\
\hline$>50$ years & 7 & 5.71 \\
\hline \multicolumn{3}{|l|}{ 3. Primary site } \\
\hline Rectum & 8 & 6.75 \\
\hline RSJ and Sigmoid colectomy & 3 & 6 \\
\hline \multicolumn{3}{|l|}{ 4.Type of surgery } \\
\hline Anterior resection and sigmoid colectomy & 7 & 8.4 \\
\hline Abdominoperineal resection & 4 & 4.5 \\
\hline \multicolumn{3}{|l|}{ 5. Primary tumour stage } \\
\hline TO & 1 & 0 \\
\hline T1 & 1 & 0 \\
\hline T2 & 3 & 7.3 \\
\hline T3 & 3 & 7.6 \\
\hline T4 & 2 & 10 \\
\hline \multicolumn{3}{|l|}{ 6. Tumour Size } \\
\hline$\leq 5 \mathrm{~cm}$ & 6 & 5.3 \\
\hline$>5 \mathrm{~cm}$ & 5 & 8 \\
\hline \multicolumn{3}{|l|}{ 7. Tumour Grade } \\
\hline No & 1 & 0 \\
\hline Low Grade & 9 & 7.2 \\
\hline High Grade & 1 & 7 \\
\hline \multicolumn{3}{|l|}{ 8. Tumour site perforation } \\
\hline ABSENT & 9 & 5.77 \\
\hline PRESENT & 2 & 10 \\
\hline \multicolumn{3}{|l|}{ 9. Specimen length } \\
\hline$<20 \mathrm{~cm}$ & 4 & 5.25 \\
\hline$\geq 20 \mathrm{~cm}$ & 7 & 7.29 \\
\hline \multicolumn{3}{|l|}{ 10. Neoadjuvant therapy } \\
\hline Yes & 7 & 6.7 \\
\hline No & 4 & 6.25 \\
\hline
\end{tabular}




\section{Discussion}

Inadequate lymph node examination is a common pitfall in the pathologic staging of colorectal cancer. This staging defect causes a great clinical challenge for predicting the prognosis and determining adjuvant treatments. The standard management for patients with stage III colorectal cancers includes curative surgery combined with adjuvant therapy. Retrieval of inadequate lymph node examination may classify patients with actual stage III disease as stage I or II diseases. They might thus be deprived of optimal treatment. ${ }^{[3]}$ Studies have shown that conventional pathologic examination of increased numbers of lymph nodes is itself associated with an increased survival advantage in stage II disease, indicating optimal dissection by pathologists. ${ }^{[3,4]}$

In 2007, the National Quality Forum listed the presence of at least 12 lymph nodes in a surgical resection among the key quality measures for colon cancer in the United States. If less than 12 lymph nodes are found, re-grossing is recommended. ${ }^{[3]}$

In the multidisciplinary tumour board meeting, conducted every month, if the lymph node yield is less than 12, surgical colleagues show their dissatisfaction.

The NCCN recommends that if initially $<12$ lymph nodes are found, then the pathologist should go back again to the specimen and submit more tissue. If still, the yield is inadequate, then a comment should be included in the report stating about the extensive search. ${ }^{[3]}$

The number of lymph nodes retrieved from a specimen of colorectal carcinoma may vary. Factors that can affect lymph node yield are age of the patient, obesity, location of the tumour, neoadjuvant therapy, surgical technique, and pathologist's handling of the specimen. In the literature, distal colonic cancers, early-stage, age, sex, BMI, and type of operation are factors that influence the lymph node yield. ${ }^{[1-2,4-7]}$

In a study by Deodhar et al, distal colonic location, small tumour volume, early-stage, neoadjuvant or preoperative radiotherapy were factors adversely affecting the lymph node yield. In this study, 13 of the total 61 cases [21.3\%], histopathology reports mentioned specifically that a relook gross examination/or extensive search for nodes has been carried out. ${ }^{[4]}$

We had a total 11 cases with MNY less than 12 nodes, but only one [0.90\%] histopathology report mentioned specifically about the extensive search for lymph nodes. Other findings were consistent with our results.

In the review article by Ong et al and Wong S L et al, the number of lymph nodes obtained in resection specimen for colorectal cancer was associated with the length of the resected segment, patient age, tumour location and characteristics, neoadjuvant therapy. ${ }^{[2,6]}$ These findings are consistent with our study.

In a multi-centric randomized study done by Mekennamp $\mathrm{L}$ et al, factors associated with the decreased number of lymph nodes were age $>60$ years, obese patient, low invasion/depth of tumour, poor differentiation grade of the tumour, and absence of lymphoid reaction. ${ }^{[7]}$

A study by Rajput et al. showed that $92 \%$ of National Comprehensive Cancer Network [NCCN] centres had achieved a benchmark of $>12$ lymph nodes. In this study, Stage I disease, or left colon primary site all were less likely to meet the target of 12 lymph nodes. ${ }^{[8]}$ The results were comparable.

In our study, cases with high tumour grade and even cases with low tumour grade had a yield of 7 , contradicting the available literature. This may be due to the lesser number of cases having a high-grade tumour.

In the study by Chou et al, the MNY [+/- SD] was $12[+/-$ 9.3]. In their study, they concluded that patients with rectal cancer and older patients who had distally located, early colon cancer were less likely to meet the benchmark yield of 12 lymph nodes. Lymph node yield is influenced by the extent of mesenteric resection, the pathologic technique, patient age, and tumour location. Also, tumour location and radiotherapy were significant predictors of lymph node yield in their study. ${ }^{[8]}$

In our study, $57.14 \%$ of patients had rectal cancers, amongst them $20 \%$ had inadequate lymph node yield.

In the study by Leung et al., the operating surgeon and examining pathologist had a significant impact on the number of lymph node harvested whereas the factors like age, T stage, previous surgery, and surgical approach [laparoscopic vs open surgery] did not affect lymph node yield. ${ }^{[9]}$

Inthestudyby Morcosetal, preoperativechemoradiotherapy, distal rectal cancer and female sex adversely affected the lymph node yield. Although age, tumour stage and grade, type of operation, and surgical delay did not affect the number of lymph nodes removed in this study. ${ }^{[10]}$

In the study by E Orsenigo et al, higher lymph nodes counts were associated with female sex, right colon cancer, emergency surgery, pT3-T4 diseases, higher tumour size, and resected specimen length. In our study, the male gender had a relatively higher yield, other results were comparable. They concluded that multiple tumour factors and patient factors are associated with lymph node yield, but only the 
removal of at least 12 lymph nodes will reliably determine lymph node status. ${ }^{[1]}$

In a retrospective study by Shen $\mathrm{S} S$ et al, MNY decreased with increasing age. Our study had similar results. ${ }^{[12]}$

In the interesting study, Gurwalia $\mathrm{J}$ et al, concluded that retrieval of fewer than 12 lymph nodes in patients with neoadjuvant radiotherapy with or without chemotherapy should be considered as a good indicator of tumour response with better local disease control, and a good prognostic factor, rather than as a pointer of poor diligence of the surgical and pathological assessment. ${ }^{[13]}$

Fat clearance techniques using xylene, acetone and alcohol increases the yield of nodes, with an increase in positive nodes. ${ }^{[14,16,17]}$ Ex-vivo intra-arterial methylene blue injection of CRC specimens has shown an increase in lymph node yield. ${ }^{[17]}$ These techniques were not performed in our centre.

In the review study by Wood $\mathrm{P}$ et al, lymph node yield is frequently reduced in older, obese patients, male sex, right-sided cancer, large, and poorly differentiated tumours. Patient ethnicity and lower socioeconomic class may negatively influence lymph node yield. Pre-operative tumour tattooing appears to increase lymph node yield. ${ }^{[15]}$ Our results were concordant with their study, but factors like ethnicity and socioeconomic status were not studied as ours is a unicentric study.

In the study by Wright et al. found that only $25 \%$ the pathologist identified that a minimum of 12 lymph nodes is necessary for accurate designation of node negativity. Reasons like paucity of time, lack of pathologists, and inadequate length of colon or mesocolon resulted in low MNY. ${ }^{[18]}$

Limitations of our study include, unicentric study. A lower number of cases. Not mentioning of re-gross in the report. Not using any fat clearance techniques or dye.

\section{Conclusion}

The lymph node yield is influenced by various factors. Lymph nodes may be more difficult to identify in specimens from patient who are elderly, with APR type of surgery, lesser tumour load (size and stage), absence of perforation at the tumour site, shorter specimen length and after neoadjuvant therapy.

If fewer than 12 Lymph nodes are found, further investigation by re-grossing the specimen for additional lymph nodes by another pathologist should be considered and if still inadequate it should be mentioned in the report.

MNY can be increased by investment of time and good technical skill of lymph node dissection, thereby accurately staging patients \& making them receive appropriate treatment.

\section{Acknowledgements}

Management of Saifee hospital, Mumbai and Histopathology department, Saifee hospital.

\section{Funding}

No funding

\section{Competing Interests}

None.

\section{Reference}

1. College of American Pathologists. Protocol for Examination of Specimens From Patients With Primary Carcinoma of the Colon and Rectum. Version: ColoRectum 3.4.0.0. CAP; 2016. Available from: https://documents.cap.org/protocols/ cp-colon-rectum-2016-v3400.pdf. [Last assessed on: 2017 September 9]

2. Mark L H Ong and John B Schofield. Assessment of Lymph node involvement in colorectal cancer. World J Gastrointest Surg. 2016;8[3]:179-192 . [DOI: 10.4240/wjgs.v8.i3.179]

3. National Comprehensive Cancer Network [NCCN]. NCCN clinical practice guidelines in Oncology. Principles of pathologic review for colon and rectal cancer. NCCN clinical practice guidelines Version 2.2016. Available from: https:// www2.tri-kobe.org/nccn/guideline/archive/colorectal2016/ english/rectal.pdf. [Last assessed on: 2017 September 9]

4. Deodhar KK, Budukh A, Ramadwar M, Bal MM, Shrikhande $\mathrm{S}$ V. Are we achieving the benchmark of retrieving $12 \mathrm{Lymph}$ nodes in colorectal carcinoma specimens? Experience from a tertiary referral center in India and review of literature. Indian J Pathol Microbiol 2012; 55:38-42. 2

5. Shen SS, Haupt BX, Ro JY, Zhu J, Bailey HR, Schwartz MR. Number of Lymph nodes examined and associated clinicopathologic factors in colorectal carcinoma. Arch Pathol Lab Med. 2009;133[5]:781-786.

6. Wong SL. Lymph node counts and survival rates after resection for colon and rectal cancer. Gastrointestinal Cancer Research : GCR. 2009; 3[2 Suppl]:S33-35.

7. Mekenkamp LJM, van Krieken JHJM, Marijnen CAM, van de Velde CJH, Nagtegaal ID, Pathology Review Committee and the Co-operative Clinical Investigators. Lymph node retrieval in rectal cancer is dependent on many factors--the role of the tumor, the patient, the surgeon, the radiotherapist, and the pathologist. Am J Surg Pathol. 2009;33[10]:15471553.

8. Chou JF, Row D, Gonen M, Liu Y-H, Schrag D, Weiser MR. Clinical and pathologic factors that predict Lymph node yield from surgical specimens in colorectal cancer: a population-based study: A population-based study. Cancer 2010;116[11]:2560-2570. 
9. Leung AM, Scharf AW, Vu HN. Factors affecting number of Lymph nodes harvested in colorectal cancer. J Surg Res. 2011;168[2]:224-230.

10. Morcos B, Baker B, Al Masri M, Haddad H, Hashem $\mathrm{S}$. Lymph node yield in rectal cancer surgery: effect of preoperative chemoradiotherapy. Eur J Surg Oncol. 2010;36[4]:345-349.

11. Orsenigo E, Gasparini G, Carlucci M. Clinicopathological factors influencing Lymph node yield in colorectal cancer: A retrospective study. Gastroenterol Res Pract. 2019; 2019:5197914.

12. Shen SS, Haupt BX, Ro JY, Zhu J, Bailey HR, Schwartz MR. Number of Lymph nodes examined and associated clinicopathologic factors in colorectal carcinoma. Arch Pathol Lab Med. 2009;133[5]:781-786.

13. Gurawalia J, Dev K, Nayak SP, Kurpad V, Pandey A. Less than 12 Lymph nodes in the surgical specimen after neoadjuvant chemo-radiotherapy: an indicator of tumor regression in locally advanced rectal cancer? J Gastrointest Oncol. 2016;7[6]:946-957
14. Leung CAW, Fazzi GE, Melenhorst J, Rennspiess D, Grabsch HI. Acetone clearance of mesocolic or mesorectal fat increases Lymph node yield and may improve detection of high-risk Stage II colorectal cancer patients. Colorectal Dis. 2018;20[11]:1014-1019.

15. Wood P, Peirce C, Mulsow J. Non-surgical factors influencing Lymph node yield in colon cancer. World $\mathrm{J}$ Gastrointest Oncol. 2016;8[5]:466-73.

16. Cohen SM, Wexner SD, Schmitt SL, Nogueras JJ, Lucas FV. Effect of xylene clearance of mesenteric fat on harvest of Lymph nodes after colonic resection. Eur J Surg. 1994;160[12]:693-697.

17. Törnroos A, Shabo I, Druvefors B, Arbman G, Olsson H. Postoperative intra-arterial methylene blue injection of colorectal cancer specimens increases the number of Lymph nodes recovered: Methylene blue improves Lymph node recovery. Histopathology. 2011;58[3]:408-413.

18. Wright FC, Law CHL, Last LD, Ritacco R, Kumar D, Hsieh E, et al. Barriers to optimal assessment of Lymph nodes in colorectal cancer specimens. Am J Clin Pathol. 2004;121[5]:663-70.

*Corresponding author:

Deepa Nagarajan, A-2 Om Eash Kripa, Sant Janabai road, Vile Parle east, Mumbai 400057

Phone: $+918828689462,2226155178$

Email: renad4@gmail.com

Date of Submission : 03/12/2020

Date of Final Revision : 07/03/2021

Financial or other Competing Interests: None.

Date of Acceptance : 11/03/2021

Date of Publication : 30/03/202 